\title{
Templated Assembly of Nanoparticles into Continuous Arrays
}

\section{Supporting Information}

Natali Ostrovsky ${ }^{1,2}$, Guillaume Le Saux,2, Uri Argaman¹, I-Te Chen ${ }^{4}$, Timothy Chen ${ }^{3}$, Chih-Hao Chang $^{4}$, Guy Makov ${ }^{1,2}$, Mark Schvartzman ${ }^{1,2}$

${ }^{1}$ Departmemnt of Materials Engineering, Ilse Katz Institute for the Nanoscale Science and

Technology², Ben-Gurion University of the Negev, Beer-Sheva, Israel

${ }^{3}$ Department of Mechanical \& Aerospace Engineering, North Carolina State University, Raleigh, NC ${ }^{4}$ Walker Department of Mechanical Engineering, The University of Texas, Austin, TX

\section{Assembly of nanoparticles onto templates patterned with nucleation centers varied around} $x=3 d$
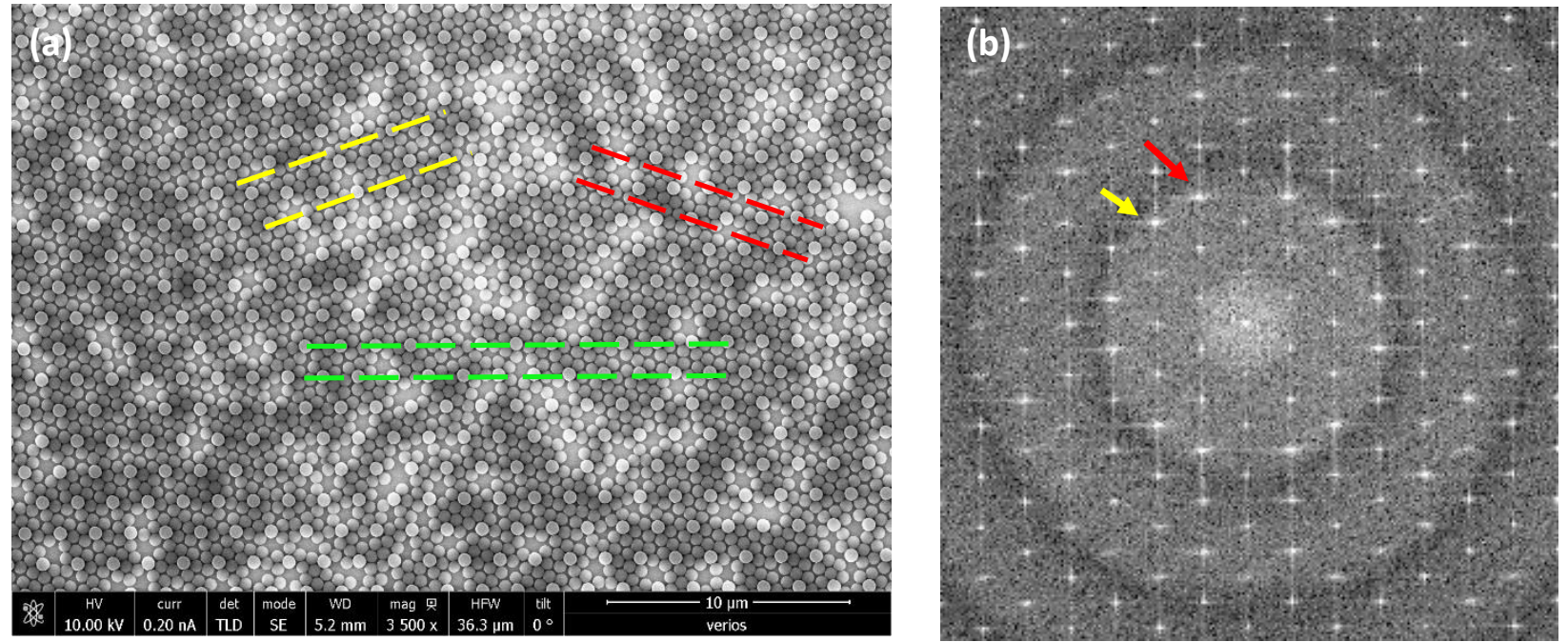

Figure S1 (a) Assembly of nanoparticles onto templates patterned with nucleation centers for $\boldsymbol{x}=\mathbf{2 . 7 d}$; (b) FFT
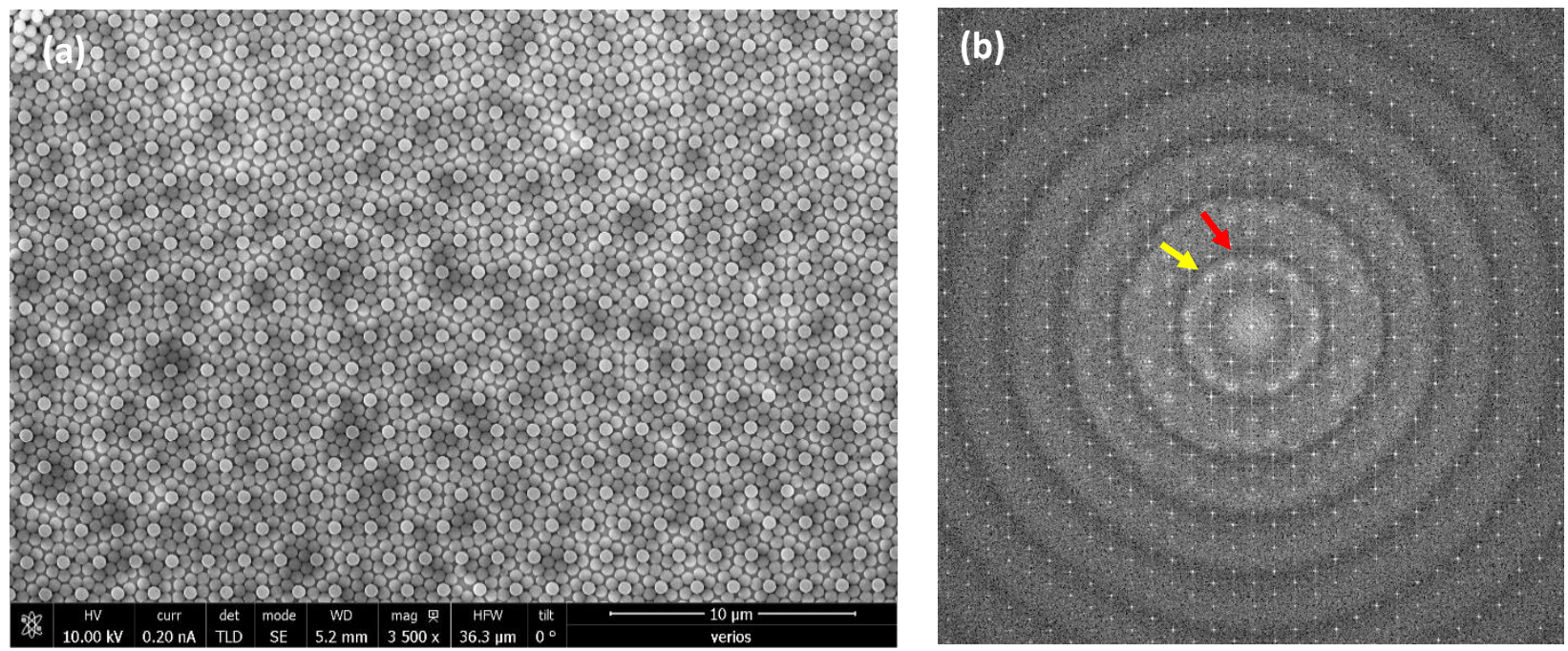
Figure S2 (a) Assembly of nanoparticles onto templates patterned with nucleation centers for $\boldsymbol{x}=\mathbf{2 . 8 d}$; (b) FFT

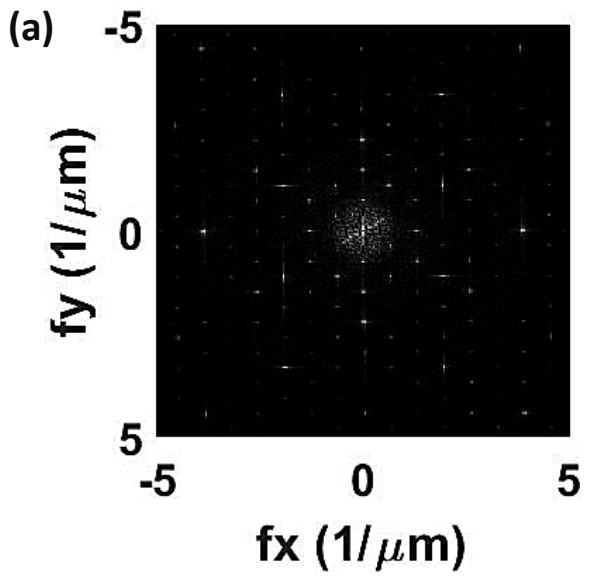

(b)

Peak Frequency: 2.23 1/um, FWHM: 0.111 1/um

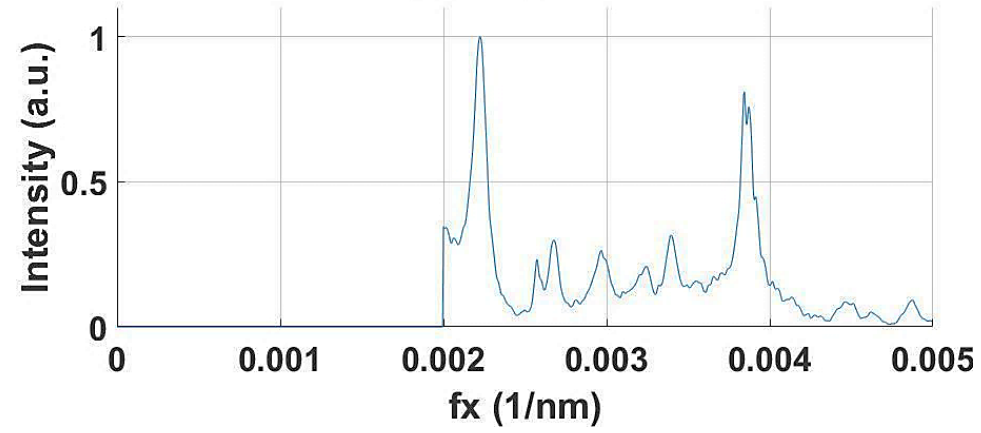

Figure S3 Example of FWHM value calculation using MATLAB (a) calculation of 2D discrete FFT of the image; (b) Measurement the nodes intensity

\section{Assembly of nanoparticles onto templates patterned with nucleation centers varied around} $\underline{x=5 d}$
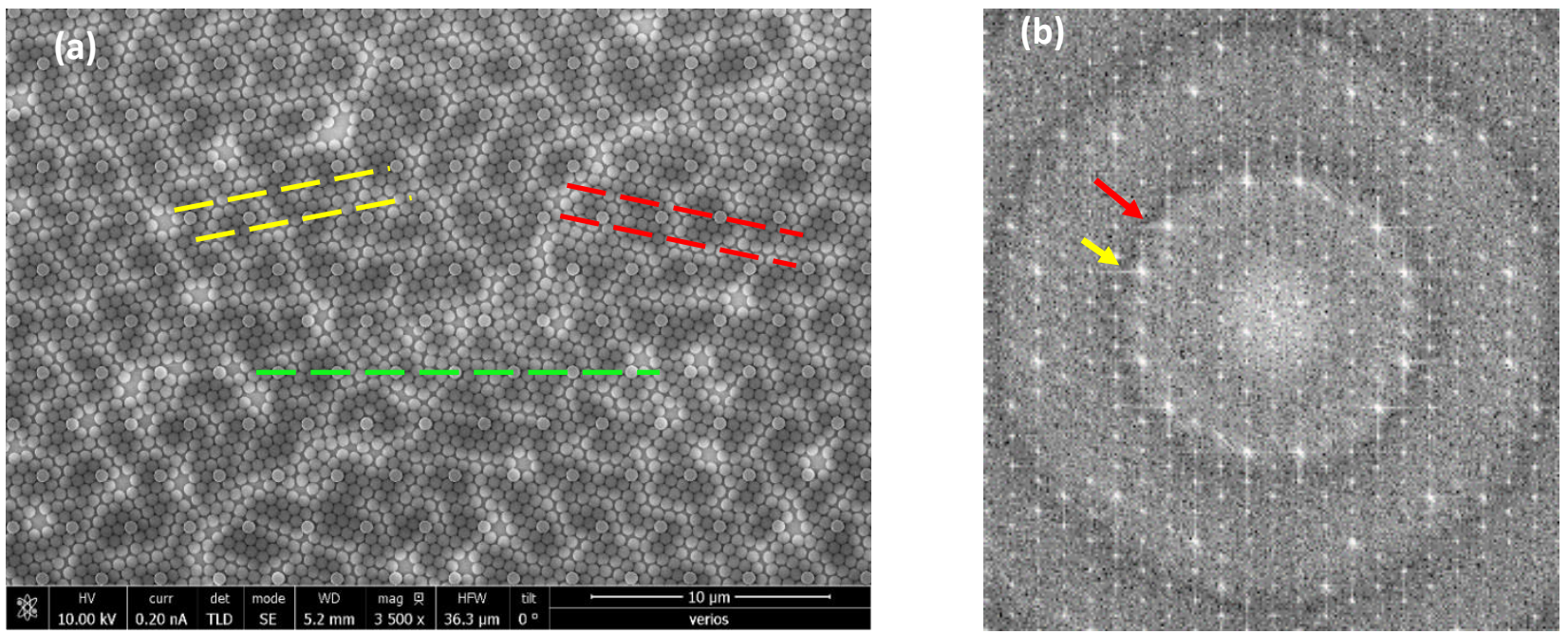

Figure S4 (a) Assembly of nanoparticles onto templates patterned with nucleation centers for $x=4.6 d$; (b) FFT 

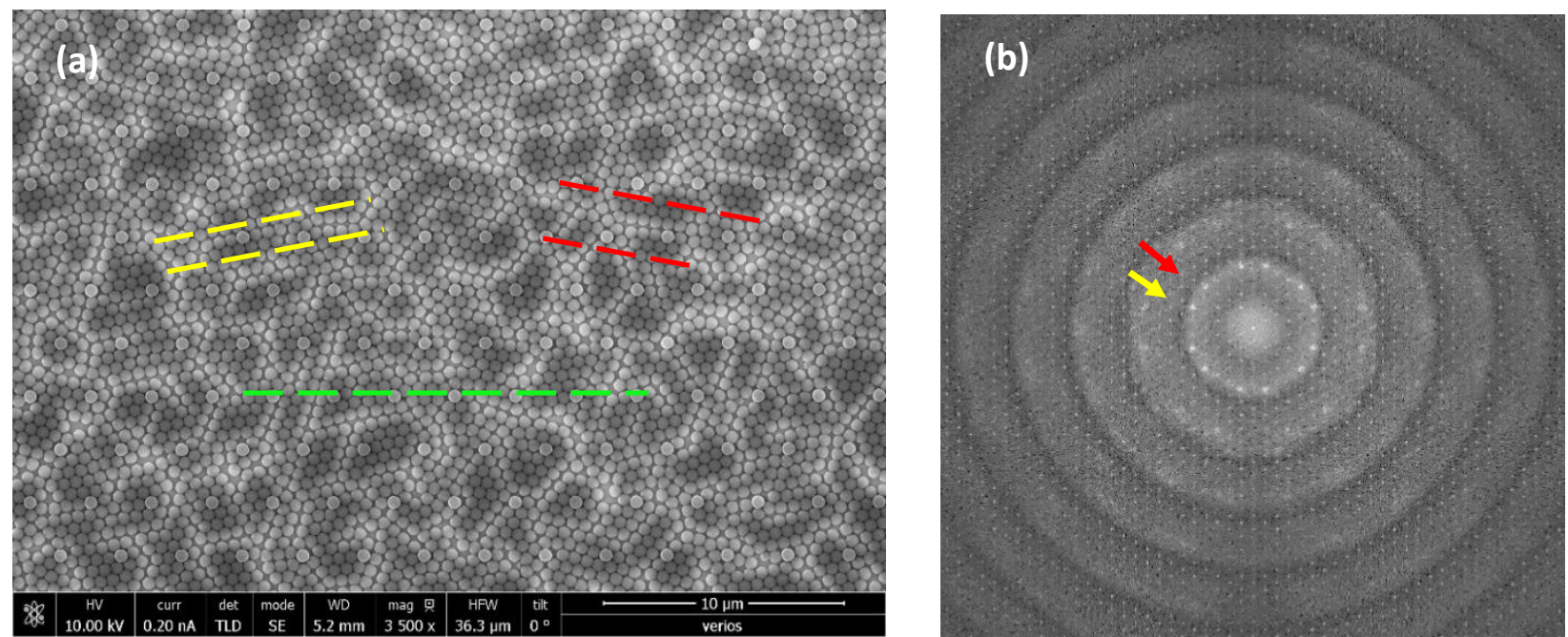

Figure S5 (a) Assembly of nanoparticles onto templates patterned with nucleation centers for $\boldsymbol{x}=4.7 d$; (b) FFT
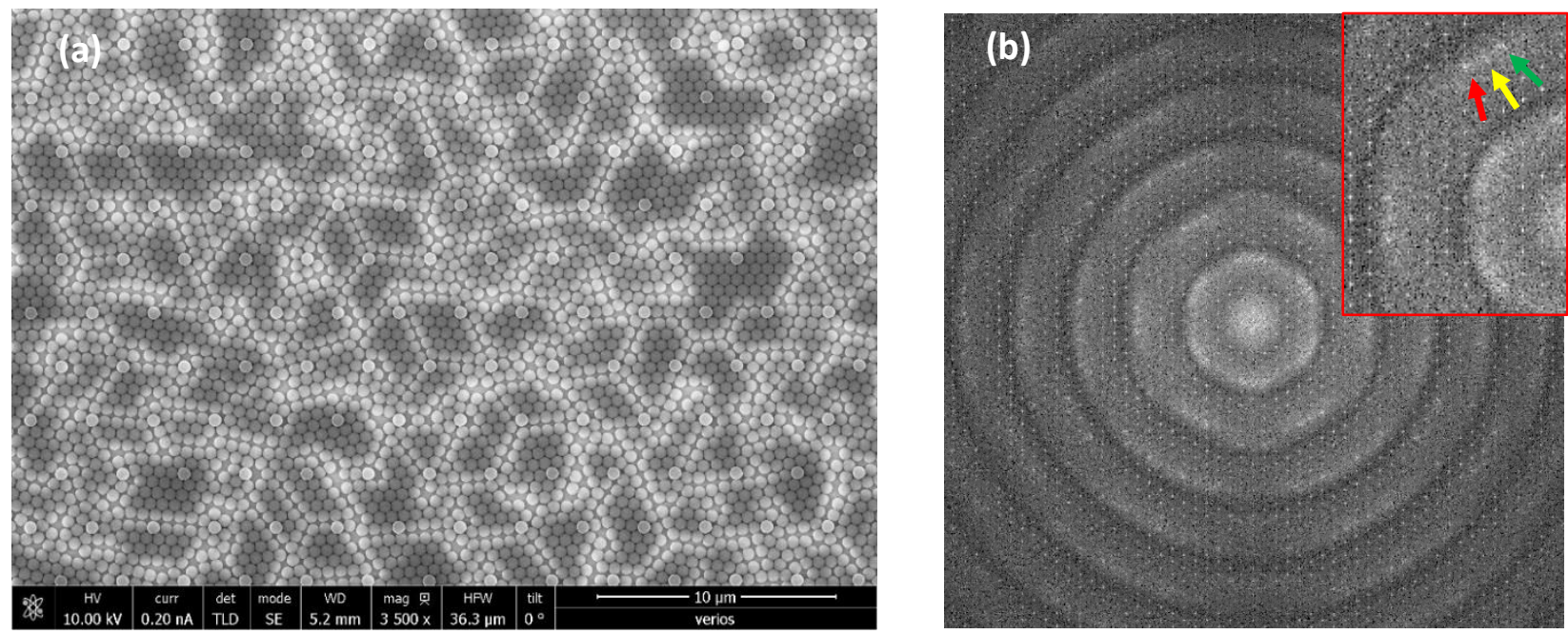

Figure S6 (a) Assembly of nanoparticles onto templates patterned with nucleation centers for $x=4.9 d$; (b) FFT 
(a)

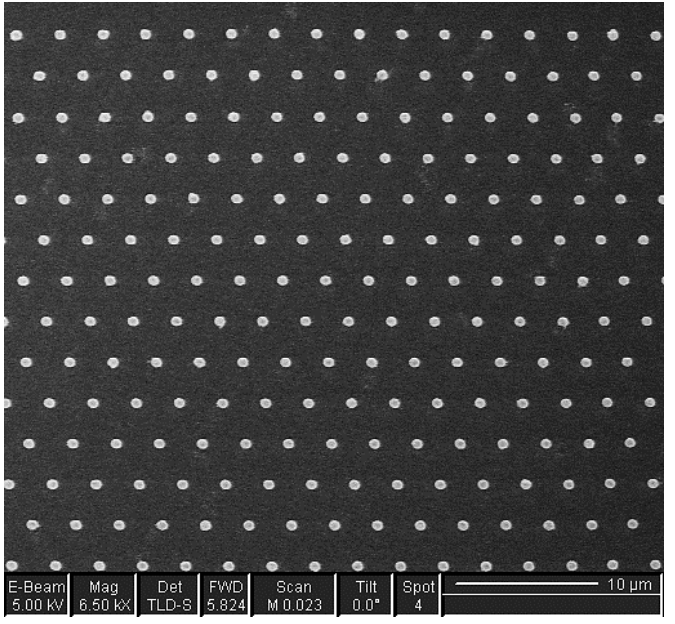

Figure S7 (a) A typical lithographic template and (b) its FFT (b)

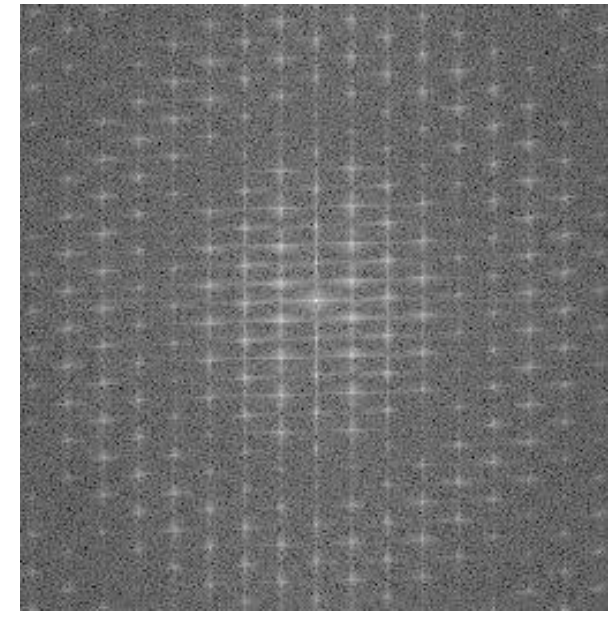


Figure 3

(d) Rotated $19^{\circ}$-unit cells

(e) Disordered assembly

(f) Closely packed assembly (g) Unpacked assembly
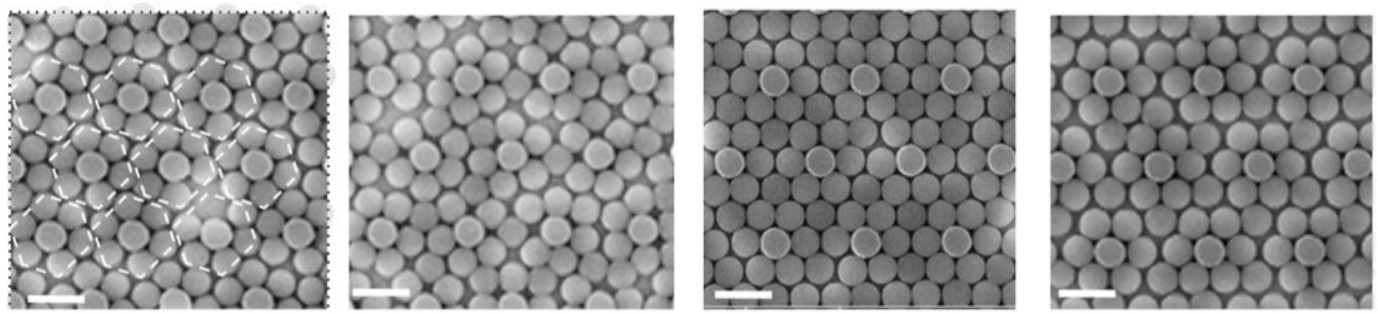

Figure 5

(d) Rotated two angles

(e) Rotated three angles

(f) Closely packed regime (g) Unpacked assembly
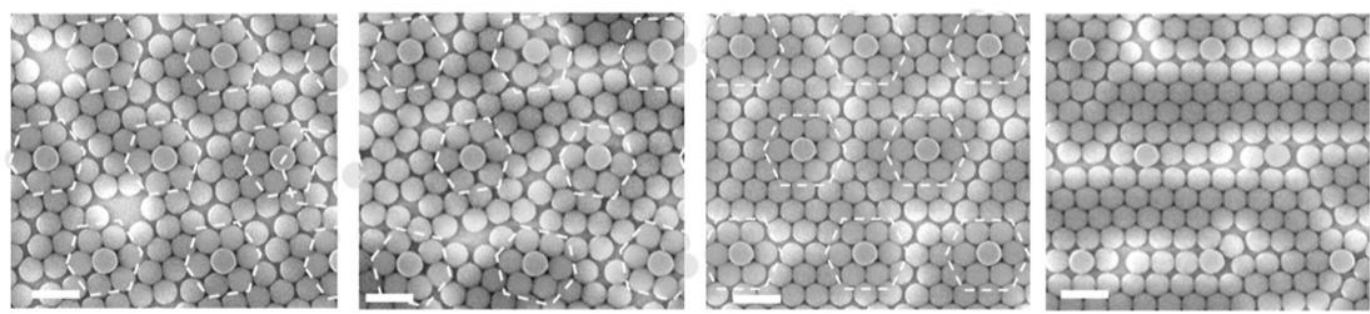

Figure 7

(d) Rotated Assembly

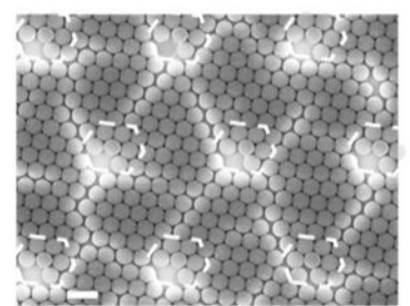

(e) Aligned Assembly

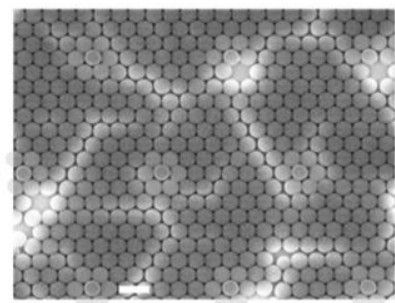

(f) Multiple microdomains with different angles

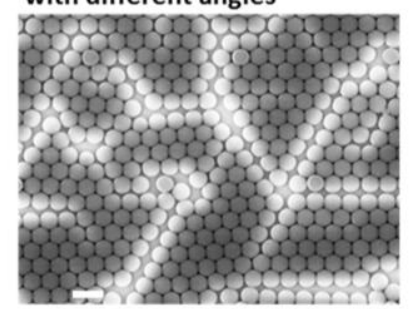

Figure S8 Black and white images of the SEM images shown in figures 3, 5 and 7. 